\title{
Influence of Torso Model Accuracy on the Noninvasive Localization of Heart Pathologies
}

\author{
Milan Tyšler ${ }^{1,2 *}$, Jana Švehlíková ${ }^{1}$, Olena Punschykova ${ }^{2,3}$ and Jana Lenková ${ }^{1}$ \\ 'Slovak Academy of Sciences, Institute of Measurement Science, Dúbravská cesta 9, 84104 Bratislava, Slovakia \\ 2 'Zzech Technical University in Praque, Faculty of Biomedical Engineering, nám. Sitnná 3105, 27201 Kladno, Cech Republic \\ 3National Technical University of Ukraine, "Kyiv Polytechnic Institute", Faculty of Biomedical Engineering, Department of Biomedical Enginee- \\ ring, 37 Prospect Peremogy, Kiev 03056, Ukraine
}

\section{BIOGRAPHICAL NOTES}

Assoc. prof. Milan Tyšler, MSc., PhD., (born in 1951) is director of the Institute of Measurement Science, Slovak Academy of Sciences in Bratislava. He obtained MSc. in Electrical Engineering from the Faculty of Electrical Engineering and Information Technology, Slovak University of Technology, Bratislava in 1974, PhD. in Measurement Science from the Institute of Measurement Theory, Slovak Academy of Sciences in 1982 and became the associate professor of the Faculty of Mechanical Engineering, Technical University in Košice in 2006. He is member of scientific boards of the FEE SUT and Slovak Institute of Metrology in Bratislava and Faculty of Biomedical Engineering, Czech Technical University in Prague, Kladno. His professional interests include modeling of the cardiovascular system and research and development of methods and devices for biosignal measurement and processing. Currently he is oriented to non-invasive methods for identification of arrhythmogenic foci and ischemic lesions in the heart. He is member of the IEEE EMBS Society, Council member of the International Society of Electrocardiology, president of the Slovak Society of BME and Medical Informatics and president of the Slovak IMEKO Committee. He is the organizer and president of several international conferences Measurement and International Congresses on Electrocardiology. He is member of the Measurement Science Review editorial board. Jana Švehlíková, MSc., PhD., (born in 1963) is a researcher in the Institute of Measurement Science, Slovak Academy of Sciences in Bratislava. She received her MSc. in Biocybernetics from the Faculty of Electrical Engineering and Information Technology, Slovak University of Technology, Bratislava in 1986 and her PhD. degree from the Institute of Measurement Science, Slovak Academy of Sciences in 2011. The topic of her dissertation was the identification of ischemic lesions from multiple lead measurement of electrical field on the human thorax. Her research interests include modeling of electrical activity of the heart, forward and inverse problem of electrocardiology and real-time measurement of biosignals.

Olena Punshchykova, MSc., (born in 1988) is currently double degree PhD. student at the Czech Technical University in Prague, Faculty of Biomedical Engineering, Department of Biomedical Technology and National Technical University of Ukraine, "Kyiv Polytechnic Institute", Faculty of Biomedical Engineering, Department of Biomedical Engineering. In 2010 she graduated with MSc. degree in Medical Devices and Systems from the National Technical University of Ukraine "Kyiv Polytechnic Institute". Topic of her dissertation thesis is myocardial excitation and mechanics of heart contraction 
correction by optimal pacing electrode positioning. She was lecturer in NTUU "KPI" in 2011 and engineer in Laboratory of Cardiology and Electrophysiology of the state establishment "National M. Amosov Institute of Cardiovascular Surgery of the Academy of Medical Sciences of Ukraine"from 2007 till 2011. Her main research area is body surface potential mapping, arrhythmias and their localization. She is an active member of Ukrainian Association of Biomedical Engineering and Technology.

Jana Lenkova, MSc., (born in 1985) is currently a PhD. student at the Slovak Academy of Sciences, Institute of Measurement Science, Department of Biomeasurements. In 2009 she graduated with MSc. degree in Biomedical Engineering from the University of Žilina, Slovakia, Faculty of Electrical Engineering. The topic of her dissertation thesis is the construction of individual torso model for the forward and inverse problem of electrocardiology using innovative approach. Her main research interests are cardiac electrical field modeling and role of individual geometry in the forward and inverse problem of electrocardiology.

\section{KEYWORDS}

ECG body surface potential mapping; inverse problem of electrocardiology; torso and heart model; noninvasive localization of heart pathology; ischemic lesion; ectopic activation.

\section{ABSTRACT}

The influence of the fidelity of an inhomogeneous torso model on the noninvasive inverse localization of selected heart pathologies was studied. It was supposed that the pathology is small enough to be represented by a single dipole. In the simulation study the accuracy of localization of an ischemic lesion was investigated. 18 ischemic lesions in the ventricles were modeled and corresponding ECGs were simulated in 7 CT-based realistic torso models. Position of each lesion represented by a single dipole was then searched by an inverse solution using different approximations of the torso and heart. If the realistic CT-based torso and heart model was used the mean error of the lesion localization was $0.7 \mathrm{~cm}$. When a common torso model adjusted to patient-specific shape using 10 anthropometric parameters with a common heart model was used, the localization error reached $3.4 \mathrm{~cm}$ and decreased to $1.0 \mathrm{~cm}$ if properly formed and rotated heart model was used. In the experimental study the site of ectopic activation was noninvasively localized in a patient with premature ventricular activity. The assessed site position was in agreement with the site determined by intracardiac mapping if realistic or adjusted common torso model with lungs and properly formed and located heart model were used. From the results of both studies it can be concluded that the use of a common torso model adjusted in concordance with patient torso dimensions and with realistic heart model can lead to acceptable accuracy of the inverse localization of the searched heart pathology.

\section{Introduction}

Recently it has been shown elsewhere $[1,2,3]$ that noninvasive cardiac imaging that uses multichannel surface ECG and proper torso and heart model for non-invasive assessment of electrophysiological state of the heart by solving the inverse problem of electrocardiology is a very promising diagnostic method. Depending on the underlying model of the cardiac electric generator it enables to identify origins of pathological electric activations, changed activation pathways or areas with changed electrical properties of the tissue. In most cases the formulation of the generator describes the whole distribution of cardiac sources in time and space, using e.g. the epi- and endocardial potentials, transmembrane potentials or dipole moments of elementary current sources. However, in some cases, if the searched pathology can be confined in space or time interval within the heart cycle, simplified source formulation is possible. In this way also a single current dipole can be used to represent e.g. initial ectopic activation, pathological activation pathway or small volume of tissue with changed repolarization $[4,5]$. The approach using single dipole as the equivalent cardiac electrical generator was used also in this study.

Besides the formulation of the source generator, the accuracy and reliability of the inverse solution is influenced by many other factors such as the number of measured ECG leads, the noise in ECG signals, the used inverse method or the fidelity of the used geometrical torso and heart model $[6,7$, 8]. This study was focused on the last mentioned factor. It is obvious that an accurate model of the heart and torso based on CT or MR imaging would be desirable. However, in clinical situations the 
whole torso imaging is usually not available in cardiac patients and approximate torso models have to be used.

We examined three types of torso models of different fidelity to find out how they affect the accuracy of the inverse localization of selected heart pathologies if the inverse method based on single dipole source formulation is used. In the computer simulation we tested noninvasive localization of small ischemic lesions while in the experimental study the localization of the site of ectopic activity was attempted in a patient with premature ventricular activation causing ventricular tachycardia that later underwent treatment in the electrophysiological lab.

\section{Method and Material}

For noninvasive inverse localization of heart pathologies that affect only a small part of the myocardial volume the corresponding cardiac electrical source can be modeled by a current dipole [9]. To assess its parameters (three coordinates and three dipole moments) generally two sets of data have to be known: the corresponding distribution of body surface potentials and geometry and electrical properties of the torso as an inhomogeneous volume conductor. In some cases it can be difficult to obtain potentials corresponding only to the searched pathology because the pathological activity is merged with electrical activities of other areas of the heart (both, healthy and pathological). In this study noninvasive localization of two pathologies will be analyzed: small ischemic lesions and sites of ectopic activation.

For the identification of a small ischemic lesion two multichannel ECG measurements should be available: the first one should represent a normal potential distribution (e.g. measured at rest without ischemia manifestation or before the ischemia occurred) and the second one should represent the pathological potential distribution with ischemia manifestation (measured after the ischemia appeared, e.g. during an exercise test). If there are no other differences between the two measurements, the difference maps obtained by subtracting the normal map from the map measured during ischemia represents only the changed electrical activity of the pathological tissue - the ischemic lesion [10]. Moreover, because the ischemic changes in cardiac cells (both, decrease and shortening of the action potentials [11]) affect the whole repolarization period, evaluation of the corresponding ST-T interval in ECG by integrating the relevant potential maps will result in a more pronounced representation of the pathological generator.

In case of an ectopic activation, the premature electrical activity of the ectopic beat starts in an unusual area of the ventricular volume and is practically the only recorded electrical activity during this part of the cardiac cycle. Hence the surface potential distribution directly reflects the pathological source. However, the ECG amplitudes during the initial phase of the ventricular activation are very small and partially immersed in noise. Therefore evaluation of a short interval of 10 to 30 ms of the initial activation (while activated area is still small enough to be represented by a single dipole) by integrating particular potential maps will again emphasize the potentials generated by the searched pathological generator and suppress the noise in ECG signals.

For all that in the rest of the study we suppose that surface potentials integrated over a proper period of time serve as the input for the inverse solution.

\subsection{Inverse Estimation of an Equivalent Integral Dipole Generator}

Integral maps over the proper time interval I were used as the input for the inverse localization of selected pathologies. Their values in body surface points are defined as

$$
i m=\int_{I} b m(t)=\mathrm{A} \int_{I} g(t)=\mathrm{A} s
$$

where $\mathrm{A}$ is a time independent transfer matrix that represents the properties of the inhomogeneous torso as a volume conductor (i.e. relations between potentials in individual surface points and multiple elementary dipoles within the myocardium), $g(t)$ is the multiple dipole generator in particular time instant $t$ of the heart activation and $s$ represents an integral generator characterizing the searched pathological electrical activity within the appropriate time interval of the heart cycle.

The equivalent integral generator EIG representing the original integral multiple dipole generator $s$ in equation (1) can be then computed as: 
$E I G=\mathrm{A}^{+} i m$

where $\mathrm{A}+$ is the pseudo-inverse of the transfer matrix $A$.

This is an ill-posed problem that in case of local pathologies (where the EIG represents only small volume of the myocardium) can be solved by approximating it by a single dipole. In the method used in this study [4] the EIG is defined only by three parameters - components of the dipole moment. To avoid solving a nonlinear problem, the other three dipole parameters - its coordinates are determined so that the dipole moments are computed for many predefined possible positions within the ventricular volume that are several millimeters apart (their spacing determines the resolution of the method). The position of the pathology is then determined as the location in which the EIG best represents the input data im and is defined by the criterion that the root mean squared difference between the map generated by the EIG and the input integral map im is minimal.

\subsection{Torso and Heart Models Used in the Inverse Calcula- tions}

To study the influence of the torso model fidelity on the result of the inverse solution three types of inhomogeneous torso models with lungs and heart cavities (their electrical conductivities were 4 times lower and 3 times higher than in the rest of the torso) were attempted in the inverse calculations:

a- Model with a common shape of torso (taken from the Dalhousie torso model [12]) adjusted according to 10 patient-specific anthropometrical parameters, lungs and a common heart model with simplified shape located in a standard position related to the position of ECG lead V2. This model corresponds to a situation when no imaging data and only basic anthropometric information on the patient torso is available.

$b$ - The same torso and lungs shape as in model " $a$ " but with a heart model properly formed and positioned to approximate the original heart shape and position. This model corresponds to cases when some imaging of the heart area is available but the torso shape can only be approximated using a few anatomical parameters.

c - True, realistic model of the inhomogeneous torso with lungs and heart. This model reflects the situation when whole torso CT or MR imaging is available for the particular patient.

\subsection{Data for the Simulation Study of Ischemia Localization}

Surface ECGs corresponding to normal activation and activation influenced by an ischemic region were simulated in inhomogeneous torso models containing lungs and heart cavities. Seven different torso models ( 6 men, 1 woman) based on real MRI scans [8] were obtained with permission and used in the study (Fig. 1). Original torso and lungs shapes were preserved; the original hearts were substituted by simplified heart models used for the simulation of activation spread.

Ventricular activation was simulated in analytically defined ventricular models based on [13]. Shape and position of each heart was adjusted for best correspondence with the original MRI scan. Normal activation as well as activation of heart with an ischemic lesion was simulated. Eighteen ischemic lesions characterized by changed repolarization with action potential shortened by $20 \%$ were modeled as a part of an ellipsoid in three areas typical for stenosis of one of the three main coronary vessels (Fig. 2): anterior - in the region supplied by the left descending artery (LAD), posterior - in the region supplied by the left circumflex artery $(L C X)$ and inferior - in the region supplied by the right coronary artery (RCA). They were located either at the endocardial or epicardial surface of
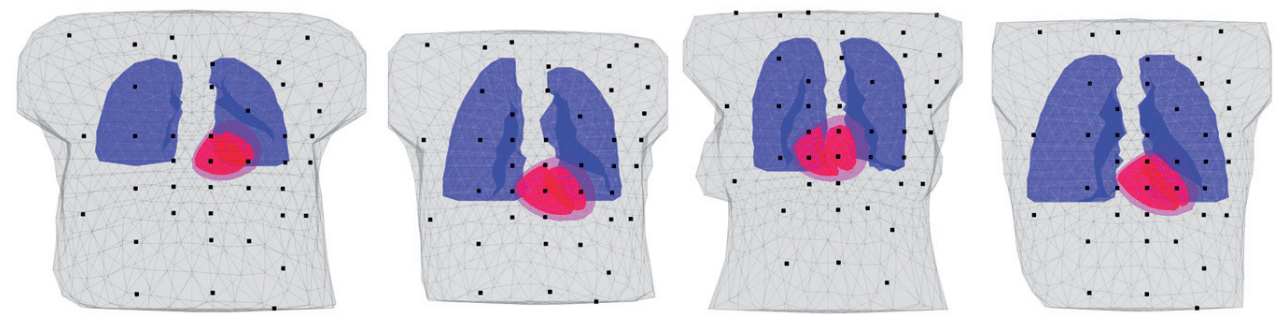

Fig. 1: Four of the 7 realistic torso models based on real MRI scans that were used in the study. 

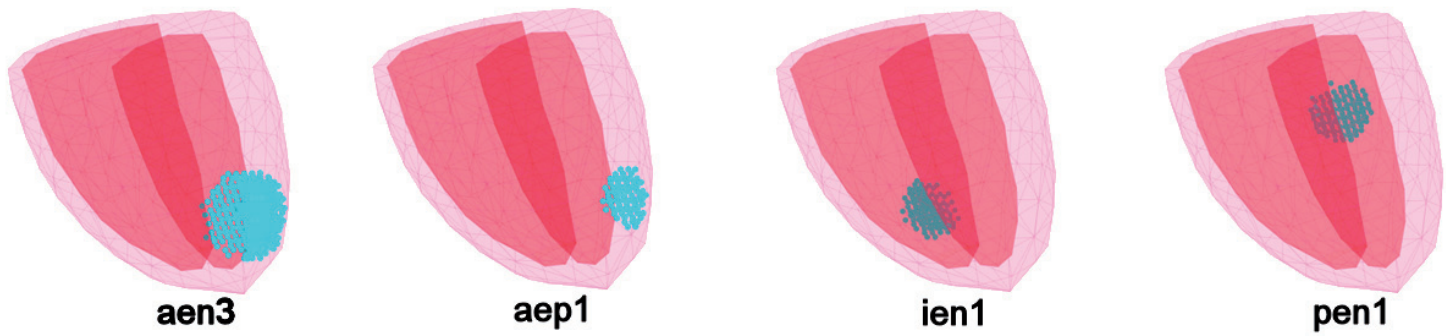

Fig. 2: Examples of the simulated ischemic lesions in the heart model: large anterior endocardial lesion (aen3), small anterior epicardial lesion (aep 1), small inferior endocardial lesion (ien 1) and small posterior endocardial lesion (pen 1).

the modeled ventricular myocardium. Three lesion sizes were modeled: small - occupying 0.5 - $1 \%$ of the ventricular volume, medium - occupying 2.5 $6 \%$ and large - occupying $8-14 \%$.

The simulated ECGs were then used as input for the inverse solution attempting to localize the position of each simulated lesion. The above described 3 types of torso models were used (Fig. 3) and their influence on the accuracy of the lesion localization was investigated.

For each torso-heart model configuration used in the inverse computations the mean lesion localization error was computed. It was defined as the distance between the gravity center of the simulated lesion and the inversely estimated position of the dipole representing the lesion.

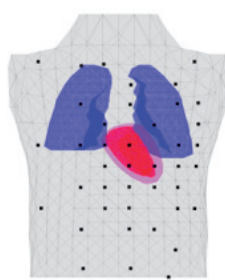

a

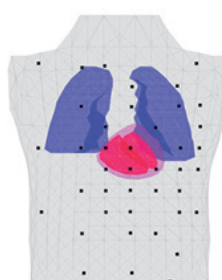

b

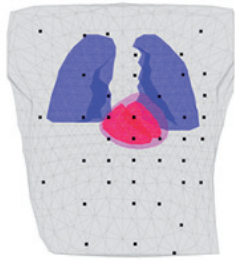

C
Fig. 3: Example of the three types of torso models used in the inverse computations for one of the 7 realistic torsos. Dots mark the positions of anterior ECG electrodes. In case c the original MRIbased torso model with simplified heart, the same as in the forward computation was used.

\subsection{Data for the Experimental Study of Ectopic Site Local- ization}

Male 57 years old patient with premature ectopic activity in ventricles causing ventricular tachycardia underwent body surface potential mapping (BSPM), computed tomography (CT) scanning and intracardiac electrophysiological study (EPS). Ten minutes BSPM was performed using the ProCardio
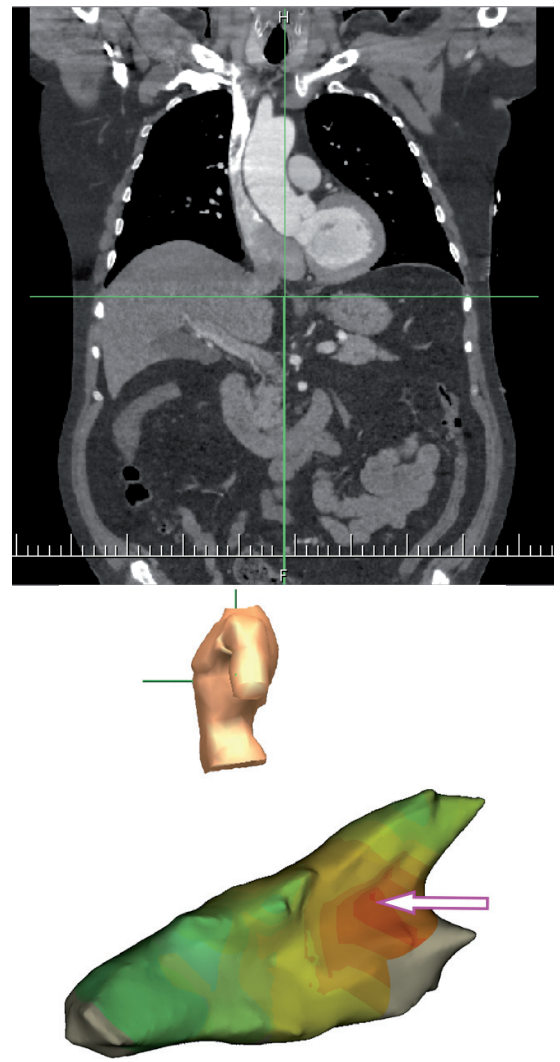

Fig. 4: Left: Frontal view of the patient's CT scan that was used for creation of the torso model and assessment of the exact positions of ECG electrodes. Right: result of the intracardiac mapping of the right ventricle. The colors (red to green) mark increasing activation times during the ectopic activation. The early activated area (marked by arrow) can be seen in septum, near the heart base.

8 system with 62 carbon electrodes placed on the torso according to the Amsterdam lead system. After BSPM the patient with fixed ECG electrodes underwent CT scanning (Fig. 4 left) using the Toshiba Aquilion ONE ${ }^{\mathrm{TM}}$ system with 320-row detector. Contrast-enhanced cardiac CT angiography scan had a slice thickness of $0.3 \mathrm{~mm}$ and a pixel size of 
$0.885 \mathrm{~mm}$. During the EPS study, St. Jude EnSite NavX ${ }^{\mathrm{TM}}$ cardiac mapping and navigation system and Bard LabSystem ${ }^{\text {TM }}$ PRO EP Recording System were used. Model of the right ventricle was created and early ectopic activity was located in the septal area near the heart base, supposing the initial site of activation in the left ventricle (Fig. 4 right). However, because of arteria femoralis sinistra occlusion the EPS was terminated without ablation. All experimental protocols were approved by the Ethic Committee of the state establishment "National M. Amosov Institute of Cardiovascular Surgery of the Academy of Medical Sciences of Ukraine" (Kyiv Ukraine) where the data collection was performed and written informed consent was obtained from the patient.

From the recorded ECG signals BSPM were computed for selected ectopic beat using the ProCardio 8 software. The inverse solution was computed from the integral map of the initial $30 \mathrm{~ms}$ of the ectopic activity.

TomoCon ${ }^{\mathrm{TM}}$ software was used for segmentation of the CT scan, construction of the torso, lungs and heart models and for assessment of accurate positions of ECG electrodes. Volumes of the lungs and ventricles were used as areas with different electrical conductivities. The points regularly spread in a $5 \mathrm{~mm}$ grid within the ventricular volume were defined as possible positions of the dipole model of the initial ectopic activation. Three types of torso models as described above were put together (Fig. 5 ) and used in the inverse computations.

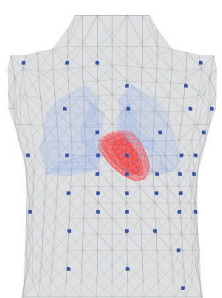

a

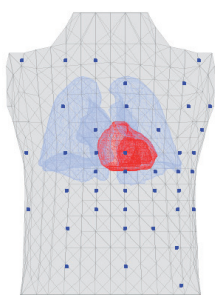

b

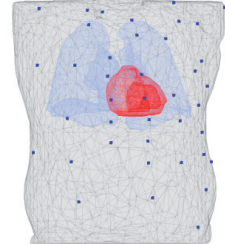

c
Fig. 5: Three types of torso, lungs and heart models as described above were used in the inverse computations. In cases " $b$ " and " $c$ " a CT-based heart model was used. Dots mark anterior electrodes.

\section{Results and Discussion}

In the simulation study the lesion localization errors were computed for all three types of torso model and for all 7 patient torsos. In each of them, 18 simulated lesions with different positions and sizes were evaluated. The mean values of the localization errors are shown in Table 1.

Table 1: Mean localization errors for all lesions for the three studied torso models.

\begin{tabular}{|l|l|}
\hline Torso model & $\begin{array}{l}\text { Mean lesion localization } \\
\text { error [cm] }\end{array}$ \\
\hline a & $3.4 \pm 1.1$ \\
\hline b & $1.0 \pm 0.7$ \\
\hline c & $0.7 \pm 0.7$ \\
\hline
\end{tabular}

As it can be seen in the table, the worst results were achieved with torso models containing the unadjusted common heart models (model "a"). Proper forming and rotation of the heart model (model "b") significantly decreased the lesion localization error to an acceptable value of $1.0 \mathrm{~cm}$ what is comparable with the intrinsic error of the method of $0.7 \mathrm{~cm}$ (model "c"). In individual cases the values of the lesion localization errors varied in relatively wide range, however, there were no significant differences between the 7 realistic torsos.

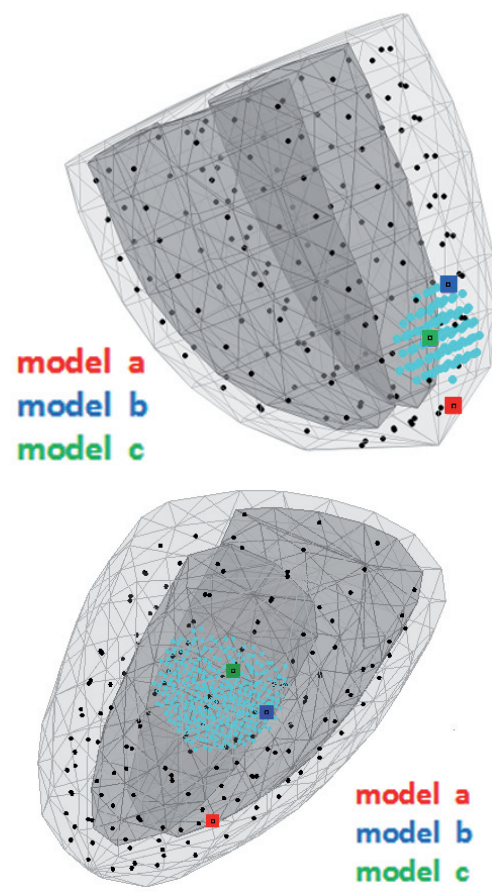

Fig. 6: Examples of simulated inverse localization of a medium anterior endocardial lesion aen2 (left) and a large posterior endocardial lesion pen3 (right) for different types of the torso models. Dots mark predefined possible positions of the equivalent dipole generator. 
For all lesion positions the mean errors were less than $1.0 \mathrm{~cm}$ if torso models " $b$ " or " $c$ " were used except the endocardial anterior lesions with the mean localization errors of $2.2 \pm 1.2 \mathrm{~cm}$ and 1.4 $\pm 1.3 \mathrm{~cm}$, respectively. Examples of the results are illustrated in Fig. 6, the localization errors correspond to values shown in Table 1.

The results of the simulation study are in correspondence with [14] where it was stated that use of adjusted standard torso shape instead of the exact one does not significantly affect the accuracy of the inverse solution.

Results of the experimental study of the noninvasive inverse localization of the site of initial ectopic activation in the patient with premature ventricular activity are shown in Fig. 7. When the common heart model was used (torso model "a") the initial site of the ectopic activation was located in the free lateral wall of the left ventricle with prevailing epi- to endocardium dipole orientation suggesting activation spread from epi- to endocardium. This result is not in concordance with the result of the EPS. Comparison of the heart models in Fig. 5 clearly indicates that the error might be due to the incorrect position of the modeled ventricular walls. When torso models "b" or " $c$ " with more realistic heart model were used, the site of initial ectopic activation was located in the posterior septum near heart base, with dipole orientation to the left ventricle and from base to apex what indicates also the prevailing direction of the activation spread and suggests that the site of the initial ectopic activation might be closer to the right ventricle. In these two cases the location of the site was found within few millimeters and is in good concordance with the EPS study, however, position of the site in the left ventricle was not clearly confirmed.

The calculations using ECG data from different ectopic beats produced slightly different results with variations of both, the position of the found initial site and the orientation of the equivalent dipole moment. However, these differences were not substantial and the results were relatively stable. The results are promising but their verification on a larger data set, possibly also with different integration intervals, is highly desirable.

\section{Conclusion}

As expected, results of the study show that accuracy of the torso and heart model can strongly influence the result of the inverse localization of heart pathologies. If CT or MRI is not available, a torso model with the shape adjusted to the patient's chest dimensions and with properly positioned, formed and oriented heart model can give acceptable accuracy of the inverse localization of small pathologies represented by single dipole. However, the simulation study also suggests that the precision of the localization may vary depending on the position of the pathology within the heart.

\section{Acknowledgments}

This study was supported by research grants 2/0131/13 from the VEGA Grant Agency, APVV0513-10 from the Slovak Research and Development Agency and SGS 13/229/OHK4/3T/17 from the SGS CVUT (Czech Republic). The authors thank prof. Maximenko, Dr. Bilynsky, Dr. Zalevsky, Dr. Stychynsky, Dr. Batsak and M. Sychyk, MSc. from the National M. Amosov Institute of Cardiovascular Surgery of the AMSU for cooperation in the clinical measurements and kind permission to use the data in this study.

\section{References}

[1] Rudy, Y. (2013) Noninvasive Electrocardiographic Imaging of Arrhythmogenic Substrates in Humans, Circulation Re-
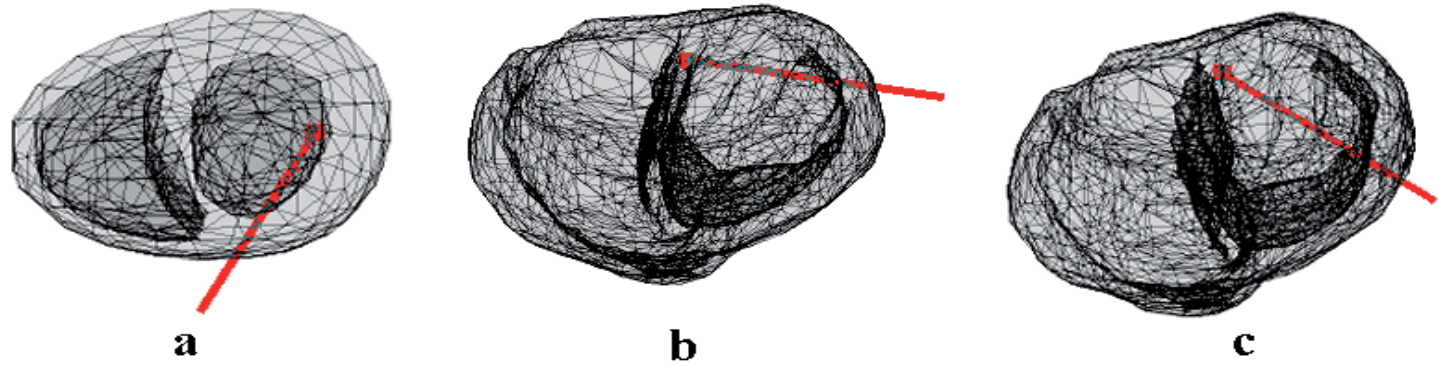

Fig. 7: Results of the inverse localization of the site of initial ectopic activation for the studied three types of the torso model. The heart models are viewed from the heart base to the apex along the long heart axis. 
search, vol. 112, 5, 863-874.

[2] Marrus, S.B., Andrews, Ch.M., Cooper, D.H. et al. (2012) Repolarization Changes Underlying Long-Term Cardiac Memory Due to Right Ventricular Pacing. Noninvasive Mapping with Electrocardiographic Imaging. Circulation - Arrhythmia and Electrophysiology, vol. 5, 4, 773-781.

[3] Haissaguerre, M., Hocini, M., Shah, A.J., et al. (2013) Noninvasive Panoramic Mapping of Human Atrial Fibrillation Mechanisms: A Feasibility Report. Journal of Cardiovascular Electrophysiology, vol. 24, 6, 711-717.

[4] Tysler, M., Kneppo, P., Turzova, M., et al. (2007) Noninvasive assessment of local myocardium repolarization changes using high resolution surface ECG mapping. Physiological Research, vol. 56, S1, S133-S141.

[5] Tysler, M., Svehlikova, J. (2013). Noninvasive finding of local repolarization changes in the heart using dipole models and simplified torso geometry. Journal of Electrocardiology, vol. 46, 4, 284-288.

[6] Huiskamp, G., VanOosterom, A. (1989). Tailored versus realistic geometry in the inverse problem of electrocardiography. IEEE Transactions on Biomedical Engineering, vol. 36, 8, 827-835.

[7] Huiskamp, GJ.M., VanOosterom, A. (1992) Heart position and orientation in forward and inverse electrocardiography. Medical \& Biological Engineering \& Computing, vol. 30, 613-620.

[8] Hoekema, R., Uijen, GJ.H., van Erning, L. et al. (1999). Interindividual variability of multilead electro-cardiographic recordings - influence of heart position. Journal of Electrocardiology, vol. 32, 2, 137-148.

[9] Lynn, M.S., Barnard, A.C., Holt, J.H. et al. (1967) A proposed method for the inverse problem in electrocardiology. Biophysical Journal, vol. 7, 6, 925-945.

[10] Trudel, M.C., Dube, B., Potse, M. et al. (2004) Simulation of QRST integral maps with a membrane based computer heart model employing parallel processing. IEEE Transactions on Biomedical Engineering, vol. 51, 8, 1319-1329

[11] Mirvis DM. Myocardial ischemia (1993). In: Mirvis DM, editor. Electrocardiology: a physiologic approach. Mosby, St. Louis, 177.

[12] Horacek, B.M., Warren, J.W., Penney, C.J. et al. (2001) Optimal electrocardiographic leads for detecting acute myocardial ischemia. Journal of Electrocardioliogy, vol. 34, S, 97-111.

[13] Szathmary, V., Osvald, R. (1994) An interactive computer model of propagated activation with analytically defined geometry of ventricles. Computers and Biomedical Research, vol. 27, 1, 27-38.

[14] Lenkova, J., Svehlikova, J., Tysler, M. (2012) Individualized model of torso surface for the inverse problem of elec- trocardiology. Journal of Electrocardiology, vol. 45, 3, 231236. 